\title{
INTELIGENCIAS MÚLTIPLES Y DIFICULTADES DE APRENDIZAJE
}

\author{
Nuria González Castellano \\ Universidad de Jaén
}

\begin{abstract}
Resumen. Cada vez más, encontramos que tanto colegios como institutos tratan de incluir metodologías más innovadoras y activas, como es trabajar de forma cooperativa, colaborativa, es decir, formando grupos de trabajo heterogéneos que ayuden al alumnado a trabajar de forma conjunta, equilibrada y así favorecer su aprendizaje. Por este motivo, en este programa de orientación e intervención psicopedagógica nos vamos a centrar en utilizar este tipo de metodología cooperativa junto con las Inteligencias Múltiples y así contribuir en el aprendizaje de los alumnos de forma más activa y constructivista. A través de los tres ámbitos de los que está compuesta la orientación educativa, Acción Tutorial, Atención a la Diversidad y Orientación Académica y Profesional, vamos a estimular las diferentes inteligencias de nuestros alumnos además de hacer ver que cualquier dificultad no tiene por qué ser un impedimento para seguir avanzando, sino todo lo contrario, tratamos de trabajar de manera inclusiva dentro del aula, ya que todos los objetivos que se proponen en este programa de intervención pueden ser conseguidos por todos los alumnos sin presentarle ninguna barrera que se lo impida.
\end{abstract}

Palabras clave: Inteligencias Múltiples, aprendizaje colaborativo-cooperativo, dificultades específicas de aprendizaje, orientación.

\section{MULTIPLE INTELLIGENCES AND LEARNING DIFFICULTIES}

\begin{abstract}
Increasingly, we find that both primary schools and secondary schools try to include more innovative and active methodologies, such as working in a cooperative or collaborative way, that is to say, forming workgroups that help students to work together and thus facilitate their learning. For this reason, in this program sychopedagogical orientation and intervention we are going to focus on using this type of cooperative methodology together with Multiple Intelligences and thus contribute in the learning process of students in a more active and constructivist way. Through the three areas of which the educational orientation is composed, Tutorial Action, Attention to Diversity and Academic and Professional Orientation, we are going to stimulate the different intelligences of our students as well as to make it clear that any difficulty is not an obstacle to move forward, But on the contrary, try to work in inclusive way in the classroom, now that all the objectives proposed in this intervention program they can be achieved by all students without showing you any barrier that prevents it is.
\end{abstract}

Keywords: Multiple Intelligences, collaborative-cooperative learning, specific learning difficulties, orientation. 


\section{Introducción}

Los orígenes de la orientación en España han sido complicados y su desarrollo en el sistema educativo fue muy lento. A partir de la Ley de Ordenación General del Sistema Educativo (LOGSE) de 1990 y la reforma educativa que esta supuso, fue cuando se consideró que la Orientación Educativa era necesaria para favorecer la educación y que formara parte de la función docente.

Más adelante, el concepto de orientación sigue apareciendo en leyes posteriores. Encontramos que la Ley Orgánica 2/2006, de 3 de mayo, de Educación prestará especial atención, en la Educación Secundaria Obligatoria, a la orientación educativa y profesional del alumnado del mismo modo que promueve las medidas necesarias para que la tutoría personal de los alumnos y la orientación educativa, psicopedagógica y profesional, constituyan un elemento fundamental en la ordenación de esta etapa. En la actual ley educativa, la Ley Orgánica 8/2013, de 9 de diciembre, para la mejora de la calidad educativa, también se manifiesta la importancia de la orientación educativa.

Por otra parte, en el Decreto 213/1995, de 12 de septiembre de 1995, por el que se regulan los equipos de orientación educativa, se señala que la orientación educativa queda dividida en tres campos, estos son, Acción Tutorial, Atención a la Diversidad y Orientación Vocacional y Profesional.

Así pues, para aplicar estos tres ámbitos de la orientación, vamos a fomentar las Inteligencias Múltiples del alumnado a través del uso de metodologías activas, en este caso a través de un tipo de aprendizaje cooperativo-colaborativo. Utilizar este tipo de metodología es debido a que nos ayuda a que los alumnos descubran cuáles son sus destrezas predominantes y cuáles son las destrezas donde tienen menos habilidad y así poder compensar unas con otras. Además, de esta manera, podemos formar grupos de trabajo heterogéneos y equilibrados que favorezcan el trabajo en equipo de los alumnos, pudiendo así ayudarse mutuamente.

Como podemos observar, donde más se utiliza la Teoría de las Inteligencias Múltiples es en niveles educativos iniciales como infantil y primaria, aunque cada vez más podemos encontrar a profesores de enseñanzas medias, como la Educación Secundaria Obligatoria, que son más innovadores y buscan otras metodologías que les ayuda a motivar el proceso de enseñanza-aprendizaje de sus alumnos. Algunos centros educativos donde podemos ver que están trabajando con las Inteligencias Múltiples y para ello han diseñado proyectos que abarcan infantil, primaria y secundaria son, los colegios Divino Maestro, Marcelo Spínola de Jaén, el colegio de los Trinitarios de Córdoba, el colegio Antonio de Nebrija de Murcia, el colegio San Cristóbal de Castellón y el colegio Princesa de Asturias de Elche.

Hay que tener en cuenta que, nos enfrentamos a una sociedad cambiante, en la que los adolescentes que nos encontramos hoy en día en los institutos no son los que había hace unos diez o quince años. Con esto queremos decir, que no podemos seguir trabajando con métodos en los que prime una metodología tradicional, poco motivadora, que incremente el escaso interés por aprender que, en algunos casos, podemos encontrar en estos alumnos. Dicho esto, hemos de decir que no solo basta con tratar de buscar una forma de trabajar con los alumnos que trate de motivarlos, sino que el profesorado tiene que formarse, investigar, asesorarse, conocer a sus alumnos y a partir de ahí, empezar aplicar metodologías que los activen y fomenten su curiosidad para aprender. 
En este sentido, podemos decir que la orientación educativa es de suma importancia en los centros educativos, ya que por medio de esta y la cooperación tanto de los tutores de los alumnos como del resto del profesorado, podemos conseguir trabajar más al unísono y así poder enriquecer el aprendizaje de los alumnos. Para ello, encontramos el desarrollo de los tres ámbitos de la orientación en este programa de intervención.

En primer lugar, hacemos alusión a la Acción Tutorial; en ella pretendemos que los alumnos se conozcan a sí mismos, entre ellos, y además descubran, a través de unas sesiones de trabajo, la Teoría de las Inteligencias Múltiples y así puedan indagar sobre aquello que puedan tener mayor o menor habilidad. Además, queremos conseguir formar grupos de trabajo heterogéneos que les ayude a trabajar más compensados y equilibrados.

En segundo lugar, encontramos la unidad de Atención a la Diversidad, en ella vamos a trabajar con un alumno con dificultades específicas de aprendizaje, en este caso dislexia. Para ello, vamos a utilizar las Inteligencias Múltiples, es decir, hemos diseñado unas sesiones de trabajo en las que podemos ver como una inteligencia complementa a otra y así poder favorecer su aprendizaje y además con esta metodología de trabajo, también se trata de favorecer la inclusión del alumnado a partir del trabajo grupal.

Por último, tenemos la unidad de intervención de Orientación Académica y Profesional. Con ella pretendemos iniciar la orientación desde cursos iniciales, en este caso primero de ESO, ya que esta orientación suele iniciarse en cursos posteriores y se dan casos en los que los alumnos llegan a segundo de Bachillerato y no saben que elegir o qué hacer después de esta etapa educativa. Para ello, vamos a utilizar también la Teoría de las Inteligencias Múltiples y así conseguir que desde cursos iniciales vayan conociendo la inteligencia en la que predominan más, sean capaces de saber que asignaturas se le dan mejor o peor y además ser competentes en tomar decisiones, pues con la nueva Ley educativa, la Ley Orgánica 8/2013, de 9 de diciembre, para la mejora de la calidad educativa (LOMCE), a partir de segundo de la ESO tienen que empezar a tomar decisiones, en cuanto a las asignaturas que quieren cursar en tercero y así sucesivamente.

\section{Análisis de la literatura}

\section{Inteligencias Múltiples}

El concepto de inteligencia, a lo largo de los años, ha sufrido multitud de variaciones, como consecuencia de los cambios sociales, científicos y culturales (Matamoros, 1999).

En un principio, la inteligencia se consideró como algo más bien biológico, hereditario, que estaba dentro de la mente del individuo y que podía medirse; es decir, como una propiedad situada en la cabeza de las personas (Gardner, Kornhaber y Krechevsky, 1993).

Debido al progreso de la sociedad y el enriquecimiento del conocimiento, se fue desarrollando un concepto más preciso y determinado del concepto de inteligencia, beneficiando el enriquecimiento del conocimiento, en la preparación de las personas, siendo estas más competentes en las diferentes disciplinas (Gomis, 2007).

Por lo tanto, podemos decir que, el desarrollo armónico de la vida de las personas, el éxito social, académico, afectivo, profesional, no puede ser explicado solo a partir de una concepción de la inteligencia o solo con el funcionamiento de una adecuada estructura cognitiva (Salmerón, 2002).

En este sentido, Valera y Cruz (2006) sostienen la afirmación de Gardner (2001) de que las personas no tenemos una sola inteligencia de tipo general, medible según los tradicionales test de inteligencia, sino que ésta tiene una estructura múltiple. Por eso mismo, cada niño presenta un perfil característico de capacidades diferentes, es decir, un conjunto de 
inteligencias, y estas inteligencias pueden reforzarse gracias a las oportunidades que ofrezca la educación y a un medio rico en materiales y actividades atractivas.

Todas las inteligencias existen en todas las personas. Se puede dar el caso de que, en algunas personas, una o más de sus inteligencias pueden estar más enfatizadas o más delimitadas, pero, en todas las personas, todas las inteligencias se presentan para ser estimuladas (Antunes, 2001).

Por lo tanto, en la Teoría de las Inteligencias Múltiples, se parte de la hipótesis de que, para que los niños logren desarrollar sus múltiples capacidades intelectuales y habilidades, es importante proporcionar una adecuada estimulación en el aula y en su vida diaria (Molejón y Fernández, 2017).

Con esto queremos decir que aprendemos con múltiples inteligencias y, por lo tanto, una educación que quiere incluir a todos, debe manejar todos los lenguajes de esas Inteligencias Múltiples (De la Cruz y García, 2017).

Tal y como afirma Gardner, estas inteligencias serían ocho (Figura 1), por lo que el ser humano tendría ocho puntos diferentes de su cerebro donde se encontrarían diferentes inteligencias; siendo estas inteligencias, lo que él denomina Inteligencias Múltiples. Serían la inteligencia lingüística o verbal, la lógica-matemática, la espacial, la musical, la cinestética corporal, la naturalista y las inteligencias personales, es decir, la intrapersonal y la interpersonal (Antunes, 2001).

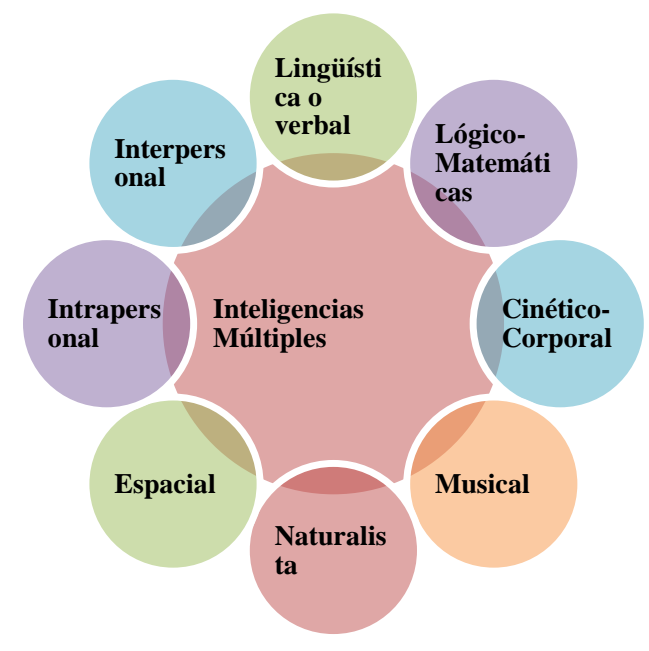

Figura 1. Inteligencias Múltiples

¿Cómo introducir las Inteligencias Múltiples en el aula?

Hay que tener en cuenta que en una clase se encuentran alumnos con diferente nivel curricular, con diferentes necesidades, capacidades, por lo que el proceso de enseñanzaaprendizaje tiene que adaptarse a esas capacidades e intereses llevar a cabo una correcta inclusión.

Para poder aplicar en el aula las Inteligencias Múltiples debemos de efectuar un estilo de enseñanza transversal y pluridisciplinar. Además, se requiere realizar una observación continua de los alumnos para poder informarnos acerca de cuál es la inteligencia en la que cada uno destaca y así poder desarrollar una metodología centrada en los alumnos que les proporcione posibilidades para mejorar en sus habilidades escolares. 
Por consiguiente, emplear en el aula las Inteligencias Múltiples, permite al docente atraer la atención de los alumnos, pues se utilizan materiales motivadores para trabajar todas las inteligencias, potenciando la autoestima, la motivación, ofreciendo un mejor clima en el aula y en la relación entre compañeros. Además, nos permite tener en cuenta los intereses, capacidades e Inteligencias Múltiples de cada uno de los alumnos; de este modo, el proceso de enseñanza-aprendizaje se puede personalizar adaptándose a sus necesidades, ya que podemos identificar aquellas áreas e inteligencias donde se demande más apoyo, facilitando la atención a la diversidad en el aula, consiguiendo una integración de todo el alumnado y de esta manera, los alumnos pueden tener un mejor conocimiento de sí mismos y de su estilo de aprendizaje (Molejón y Fernández, 2017).

De este modo, en este estilo de enseñanza es necesario promover el aprendizaje colaborativo-cooperativo, como por ejemplo el Aprendizaje Basado en Proyectos, el Proyecto Spectrum, Key School, Art Propel.

\section{Dificultades Específicas de Aprendizaje}

Como podemos ver, tanto en la Ley Orgánica 8/2013, de 9 de diciembre, para la mejora de la calidad educativa (LOMCE) como en la Ley 17/2007, de 10 de diciembre, de Educación de Andalucía (LEA), las dificultades de aprendizaje son consideradas necesidades específicas de apoyo educativo, pues precisan una atención educativa diferente a la ordinaria.

Por otro lado, encontramos que las Instrucciones del 8 de marzo de 2017, de la Dirección General de Participación y Equidad, por las que se actualiza el protocolo de detección, identificación del alumnado con necesidades específicas de apoyo educativo y organización de la respuesta educativa, consideran que se dan dificultades específicas de aprendizaje cuando:

El alumnado requiere por un periodo de su escolarización o a lo largo de toda ella, una atención educativa diferente a la ordinaria por presentar desórdenes significativos en los procesos cognitivos básicos implicados en los procesos de aprendizaje, que interfieren significativamente en el rendimiento escolar y en las actividades de la vida cotidiana del alumno y que no vienen determinados por una discapacidad intelectual, sensorial o motórica, por un trastorno emocional grave, ni por falta de oportunidades para el aprendizaje o por factores socioculturales.

De esta manera, coincidiendo con Monedero (1989), Pérez (2002) y Aguilar (2011), un alumno presenta dificultades específicas de aprendizaje cuando le cuesta realizar adecuadamente ciertas actividades de tipo académico como leer, escribir, multiplicar, sumar o restar, dando lugar a que su rendimiento académico sea inferior al esperado.

En cuanto a los tipos de dificultades específicas de aprendizaje, según las Instrucciones del 8 de Marzo 2017, de la Dirección General de Participación y Equidad, por las que se actualiza el protocolo de detección, identificación del alumnado con necesidades específicas de apoyo educativo y organización de la respuesta educativa y Llanos (2006), las podemos clasificar en cuatro grupos:

- Dificultades específicas en el aprendizaje de la lectura de la escritura o dislexia

- Dificultades específicas en el aprendizaje de la escritura o disgrafía

- Dificultades específicas en el aprendizaje de la escritura o disortografía

- Dificultades específicas en el aprendizaje del cálculo o discalculia 


\section{Inteligencias Múltiples y Dificultades Específicas de Aprendizaje}

Observamos cómo en muchas aulas hay armonía, bienestar, aprendizaje de todos, mientras en otras hay tensión, sufrimiento, discriminación; emociones que afectan tanto a los alumnos como a los profesores, generando un clima en el que el aprendizaje es posible para todos o solo para unos pocos (De la Cruz y García, 2017).

Por esa razón, cuando identificamos a un niño con dificultades específicas de aprendizaje hay que tener en cuenta que sus dificultades no tienen que estar solo relacionadas con su capacidad de aprendizaje, sino con las estrategias que estemos utilizando con ellos en ese momento (Ugalde, s.f.).

Por lo tanto, los docentes tienen que tener en cuenta que deben de convertirse en investigadores de su propia práctica, diseñando actividades donde todos sus alumnos puedan tener éxito y así forjarlos de experiencias positivas y puedan estar vinculados con el proceso de enseñanza aprendizaje, proporcionándoles medios para que puedan descubrir y comprender lo que trabajan, siendo esta la mejor motivación que puedan manejar (De la Cruz y García, 2017).

Con lo cual, la intervención educativa con alumnos con dificultades debería ampliar su perspectiva y no centrarse únicamente en el déficit, sino también en las fortalezas y habilidades. Por ello, sería apropiado repensar el enfoque de trabajo y no desaprovechar las habilidades y talentos que los niños con dificultades pueden atesorar (González, 2014).

Todas las personas contamos con una serie de inteligencias, que dependiendo de cuándo y cómo sean estimuladas nos va a influir, por ejemplo, en la forma de enfrentarnos a la vida, de percibir información, de relacionarnos con los iguales, de adquirir aprendizajes. Por lo tanto, los niños deben de tener una estimulación temprana para potenciar toda la gama de habilidades que poseen, considerando la inteligencia como un concepto amplio, ligado al interés de cada niño y apuntando hacia su satisfacción personal (Ugalde, s.f.).

Puesto que los individuos se diferencian en la intensidad de estas inteligencias y en las formas en que recurre a esas mismas inteligencias y se las combina para llevar a cabo diferentes labores, para solucionar problemas diversos y progresar en distintos ámbitos, decimos que las personas aprenden, representan y utilizan el saber de muchos y diferentes modos. Estas diferencias retan al sistema educativo, ya que este supone que todo el mundo puede aprender las mismas materias del mismo modo y que basta con una medida uniforme y universal para poner a prueba el aprendizaje de los alumnos (Andrade, 2009).

Para finalizar, si el profesorado planteara actividades teniendo en cuenta todas las Inteligencias Múltiples y no tan solo las más relacionadas con las áreas instrumentales, se enriquecerían las situaciones de enseñanza-aprendizaje y se flexibilizaría la intervención educativa, adaptándose a las necesidades individuales de cada alumno. De este modo, cuando el profesorado plantea este tipo de actividades tiene más tiempo para atender de forma individualizada al alumnado con Necesidades Específicas de Apoyo Educativo, ya que el resto de alumnado es más autónomo para realizar las actividades debido a que son más conscientes de los diferentes mecanismos de ayuda que tienen a su alrededor, como es la búsqueda individual de información, consulta entre iguales, al docente, y la correcta forma de utilizarlos (Huelmo y Fernández, 2017). 


\section{Método}

\section{Programa de Orientación e Intervención Psicopedagógica}

\section{Participantes}

Este programa de intervención va dirigido a los alumnos de primero de la ESO, en la hora semanal de tutoría en el aula ordinaria del grupo, cada clase consta de 25 alumnos. Aunque el programa está diseñado por el orientador del centro, cada unidad va a ser impartida por el tutor, aunque en la unidad de atención a la diversidad contará con la ayuda de la maestra de Pedagogía Terapéutica (PT) en algunas sesiones de trabajo, puesto que en una clase de $1^{\circ}$ de la ESO, encontramos a un alumno que presenta dislexia y en ciertas actividades, que están adaptadas a su nivel curricular, va a necesitar el apoyo de la PT.

\section{Planificación y diseño del programa}

El objetivo general de este programa de orientación e intervención psicopedagógica es trabajar las tres áreas que constituyen la Orientación Educativa, como son la Acción Tutorial, la Atención a la Diversidad y la Orientación Académica y Profesional a través de las Inteligencias Múltiples. Para ello, en la unidad de intervención de la Acción Tutorial vamos a emplear metodologías activas, como el trabajo cooperativo-colaborativo, para el conocimiento de cada una de las inteligencias. Por otro lado, en la unidad de intervención de la Orientación Académica y Profesional, fomentaremos el trabajo autónomo y la toma de decisiones del alumnado mediante actividades relacionadas con las Inteligencias Múltiples y por último, la unidad de intervención de la Acción Tutorial, serán trabajadas las dificultades específicas de aprendizaje, como la dislexia, a través de las Inteligencias Múltiples.

Los objetivos didácticos que hemos considerado para este programa son los siguientes:

- Fomentar y trabajar el conocimiento de las Inteligencias Múltiples.

- Potenciar el trabajo en grupo del alumnado mediante el aprendizaje cooperativocolaborativo.

- Entrenar el trabajo autónomo para así poder tomar sus propias decisiones.

- Intervenir y actuar en las dificultades específicas de aprendizaje, como es la dislexia, a través de las Inteligencias Múltiples.

Haciendo referencia a los contenidos generales que se van a trabajar en dicho programa, hemos estimado:

- El conocimiento de las Inteligencias Múltiples.

- $\quad$ El trabajo en grupo a través del aprendizaje cooperativo-colaborativo.

- La capacidad de tomar decisiones.

- La intervención de la dislexia con las Inteligencias Múltiples.

En cuanto a las competencias clave que van a ser desarrolladas con la aplicación de este programa de orientación en intervención psicopedagógica son las establecidas por la Orden ECD/65/2015, de 21 de enero, por la que se describen las relaciones entre las competencias, los contenidos y los criterios de evaluación de la educación primaria, la educación secundaria obligatoria y el bachillerato. A efectos de esta orden, estas son las siete competencias:

- Comunicación lingüística. 
- $\quad$ Competencia matemática y competencias básicas en ciencia y tecnología.

- $\quad$ Competencia digital.

- $\quad$ Aprender a aprender.

- Competencias sociales y cívicas.

- Sentido de iniciativa y espíritu emprendedor

- Conciencia y expresiones culturales.

La metodología que vamos a llevar a cabo en este programa de orientación e intervención psicopedagógica, va a ser una metodología activa, trabajando de forma cooperativa, colaborativa, es decir, a través de grupos de trabajo que ayude al alumnado a trabajar de forma conjunta y así favorecer tanto su proceso de enseñanza-aprendizaje como la inclusión de todo el alumnado.

Los recursos que vamos a necesitar van a depender de la actividad que se vaya a realizar en cada unidad de intervención, es decir, recursos materiales, espaciales y personales. Estos recursos estarán especificados dentro de la explicación de cada sesión que conforme las distintas unidades de intervención.

La temporalización del programa y cada unidad de intervención, será reflejada a través de la siguiente tabla.

Tabla 1

Temporalización de las unidades de intervención

\begin{tabular}{|c|c|}
\hline \multirow[t]{7}{*}{$\begin{array}{l}\text { Primer } \\
\text { trimestre }\end{array}$} & $\begin{array}{l}\text { Unidad de intervención para la Acción Tutorial: Nos conocemos } \\
\text { mientras trabajamos. }\end{array}$ \\
\hline & Primera sesión: Nos conocemos todos (50’). \\
\hline & Segunda sesión: Normas de convivencia (55’). \\
\hline & $\begin{array}{l}\text { Tercera sesión: Somos escritores. La inteligencia lingüística y viso- } \\
\text { espacial (55'). }\end{array}$ \\
\hline & $\begin{array}{l}\text { Cuarta sesión: Somos científicos. La inteligencia matemática y } \\
\text { naturalista }\left(50^{\prime}\right) \text {. }\end{array}$ \\
\hline & $\begin{array}{l}\text { Quinta sesión: Qué sentimos. La inteligencia musical e intrapersonal } \\
\left(50^{\prime}\right) \text {. }\end{array}$ \\
\hline & $\begin{array}{l}\text { Sexta sesión: Bailamos juntos. La inteligencia cinestética e interpersonal } \\
\left(50^{\prime}\right) .\end{array}$ \\
\hline \multirow[t]{3}{*}{$\begin{array}{l}\text { Segundo } \\
\text { trimestre }\end{array}$} & $\begin{array}{l}\text { Unidad de intervención para la Atención a la Diversidad: Jugamos con } \\
\text { las palabras. }\end{array}$ \\
\hline & $\begin{array}{l}\text { Primera sesión: 1. Jugamos con las rimas. 2. Qué nos dice la lectura. } 3 . \\
\text { Palabras escondidas. } 4 \text {. Conocemos las palabras }\left(55^{\prime}\right) \text {. }\end{array}$ \\
\hline & $\begin{array}{l}\text { Segunda sesión: 5. ¿Cuántos sonidos hay? 6. Buscamos palabras y } \\
\text { formamos números. } 7 \text {. ¿Cuántas palabras hay? } 8 \text {. Reconocemos las }\end{array}$ \\
\hline
\end{tabular}




\begin{tabular}{|c|c|}
\hline \multicolumn{2}{|c|}{\begin{tabular}{l|l} 
& figuras (50’). \\
\cline { 2 - 2 }
\end{tabular}} \\
\hline & $\begin{array}{l}\text { Tercera sesión: } 9 . \text { Textos incompletos. 10. Sopa de letras. 11. Palabras } \\
\text { inventadas. 12. Buscar las que son diferentes (55'). }\end{array}$ \\
\hline & $\begin{array}{l}\text { Cuarta sesión: 13. ¿Qué es lo que suena? 14. Cualidades del sonido } \\
\left(40^{\prime}\right) \text {. }\end{array}$ \\
\hline & Quinta sesión: 15. Cuidamos el medio ambiente (50'). \\
\hline & $\begin{array}{l}\text { Sexta sesión: 16. ¿Qué escondes en la bolsa? 17. Formamos palabras } \\
\text { nuevas }\left(50^{\prime}\right) .\end{array}$ \\
\hline & Séptima sesión: 18. Autobiografía. 19. Mi mapa autobiográfico (45’). \\
\hline \multirow[t]{4}{*}{$\begin{array}{l}\text { Tercer } \\
\text { trimestre }\end{array}$} & $\begin{array}{l}\text { Unidad de intervención para la Orientación Académica y Profesional: } \\
\text { ¿Cuál es mi inteligencia? }\end{array}$ \\
\hline & Primera sesión: 1. Qué inteligencia se me da mejor (50’). \\
\hline & $\begin{array}{l}\text { Segunda sesión: 2. Asignaturas preferidas. 3. Dónde obtengo mejores } \\
\text { resultados }\left(50^{\prime}\right) \text {. }\end{array}$ \\
\hline & Tercera sesión: 4. Averiguamos las profesiones y las inteligencias (50’). \\
\hline
\end{tabular}

Nota: Fuente: elaboración propia

\section{Unidades de intervención}

\section{Acción tutorial}

Esta unidad de intervención se va a llevar a cabo a través de seis sesiones formadas por varias actividades cada una. Su principal objetivo consiste en dar a conocer cada una de las ocho Inteligencias Múltiples y para ello vamos a implantar metodologías activas como el trabajo cooperativo-colaborativo.

De esta manera ayudamos al alumnado al conocimiento de sí mismo y al resto de compañeros, a descubrir conocimientos nuevos, como son las Inteligencias Múltiples, así como la afinidad que pueden tener con cada una de ellas y formar grupos heterogéneos y equilibrados que posteriormente pueden ser utilizados para trabajar en otras materias.

En esta unidad también puede participar el alumno que presenta dislexia, ya que puede acceder a la realización de las actividades con la ayuda de la maestra de Pedagogía Terapéutica, con el fin de conseguir una completa inclusión del alumnado.

Ejemplo de algunas de las actividades que se llevarán a cabo en esta unidad de intervención:

En la tercera sesión los alumnos van a trabajar con inteligencias combinadas y así ir descubriendo por un lado, de qué se trata la Teoría de las Inteligencias Múltiples y por otro, cual es la inteligencia donde tienen más afinidad. Para comenzar, esta tercera sesión se va a centrar en la inteligencia lingüística y viso-espacial, trabajando la imaginación, la expresión oral, escrita y la transmisión de sentimientos. 
Tabla 2

Actividad 3 de la tercera sesión

Actividad 3: Somos escritores. La inteligencia lingüística y viso-espacial Temporalización

- 40 minutos para la elaboración del poema y del dibujo y 15 minutos para la exposición en grupo.

\section{Objetivo/s específicos}

- Elaborar, a través de las terminaciones dadas, un poema de cuatro versos.

- Expresar mediante un dibujo los sentimientos que les transmite el poema al leerlo.

- Saber explicar a los compañeros el dibujo que han representado.

- Trabajar la inteligencia lingüística y viso-espacial.

\section{Contenidos}

- Comprensión de las terminaciones dadas y elaboración de un poema de cuatro versos.

- Interpretación de los sentimientos manifestados con la lectura del poema, mediante la elaboración de un dibujo.

- Explicación de la representación del dibujo a los compañeros.

- Conocimiento de la inteligencia lingüística y viso-espacial.

\section{Desarrollo de la actividad}

En esta actividad van a estar organizados en pequeños grupos. En la pizarra el tutor va a escribir cuatro terminaciones que van a rimar entre sí y cada grupo va a tener que elaborar un poema de cuatro versos. Una vez que hayan elaborado el poema, tendrán que expresar, mediante un dibujo común, lo que les transmite cuando lo leen. De esta forma se está trabajando la inteligencia lingüística ya que tienen que elaborar frases que tengan sentido entre ellas y además buscar que rimen, después con la representación del dibujo visualizan lo que sienten al leer el poema, por ello también están trabajando la inteligencia viso-espacial.

Tras finalizar esta parte de la actividad, tendrán que exponerlo ante los compañeros de la clase, es decir, tendrán que recitar el poema y además explicar la representación del dibujo que se trata del reflejo de sus sentimientos.

Antes de llevar a cabo esta actividad en clase, la maestra de Pedagogía Terapéutica puede trabajarla con el alumno que presenta dislexia previamente para practicar la realización de rimas y así cuando se realice en clase pueda integrarse mejor en la actividad.

\section{Recursos y materiales}

- Folios, lápices, ceras de colores.

Criterios de evaluación

- Comprende el significado de las palabras para la elaboración del poema.

- Interpreta los sentimientos a través de la realización de un dibujo.

- Sabe expresar a los compañeros la representación del dibujo.

- Conoce la inteligencia lingüística y viso-espacial. 
Inteligencias múltiples y dificultades de aprendizaje

\begin{tabular}{c|c}
\hline Rol del profesor & Organización del espacio \\
\hline$\bullet$ Guía-orientador. & $\bullet$ Grupos pequeños. \\
\hline
\end{tabular}

Nota: Fuente: elaboración propia

\section{Atención a la diversidad}

En esta unidad de intervención, relacionada con la atención a la diversidad, nos vamos a centrar en las dificultades específicas de aprendizaje, concretamente en la dislexia, haciendo hincapié en la inclusión de todos los alumnos en el aula a través de actividades heterogéneas.

Esta intervención va a consistir en trabajar la dislexia desde las Inteligencias Múltiples a través de actividades transversales. Dichas actividades van a estar todas combinadas, es decir, partiendo en la primera sesión de la inteligencia lingüística y esta combinada, en el resto de sesiones, con otra inteligencia. La unidad va a estar compuesta por siete sesiones.

Finalmente, con esta unidad de intervención, lo que pretendemos es poder trabajar la dislexia desde metodologías activas que potencien el interés de este alumnado por aprender y así poder reducir el abandono escolar de estos discentes.

Ejemplo de algunas de las actividades que se llevarán a cabo en esta unidad de intervención:

La segunda sesión va estar compuesta por cuatro actividades, aunque en esta solo nos vamos a centrar en la Inteligencia Lingüística combinada con la Inteligencia Matemática para solventar las dificultades de comprensión de problemas matemáticos.

Tabla 3

Actividad 2 de la segunda sesión

\section{Temporalización}

\section{Actividad 2: Buscamos palabras y formamos números}

- 25 minutos.

\section{Objetivo/s específicos}

- Ordenar las palabras.

- Indicar el número de sílabas que tiene cada palabra.

- Descomponer en unidades de millar, centenas, decenas y unidades el número inventado.

\section{Contenidos}

- Ordena las palabras.

- Indica el número de sílabas que tiene cada palabra.

- Descompone en unidades de millar, centenas, decenas y unidades el número inventado.

\section{Desarrollo de la actividad}

Este ejercicio está compuesto por diferentes apartados (Anexo 1), ver ejemplo:

- Buscar las palabras escondidas, solo puedes eliminar una sílaba.

- Indicar el número de sílabas que tiene cada palabra. 
- Formar números, con el número de sílabas que nos da cada grupo de palabras.

- Descomponer el número formado en unidades de millar, centenas, decenas y unidades.

- Al ser una actividad más compleja se puede realizar con todo el grupo de clase.

\section{Recursos y materiales}

- Lápiz, goma y anexo 1.

\section{Criterios de evaluación}

- Ordena correctamente cada sílaba que compone la palabra.

- Identifica el número de sílabas que forma la palabra.

- Descompone cada número en unidades de millar, centenas, decenas y unidades.

\begin{tabular}{c|c}
\hline Rol del profesor & Organización del espacio \\
\hline $\begin{array}{l}\text { Orientador y guía, asegurándose que la } \\
\text { actividad la realiza adecuadamente. }\end{array}$ & $\bullet \quad$ Aula ordinaria. \\
\hline
\end{tabular}

Nota: Fuente: elaboración propia

\section{Orientación Académica y Profesional}

Esta Unidad de Intervención, propuesta para la Orientación Académica y Profesional, está compuesta por tres sesiones. Se llevará a cabo por medio de las Inteligencias Múltiples, ya que se pretende que el alumnado se conozca así mismo, pueda reflexionar sobre sus preferencias y sea capaz de tomar sus propias decisiones.

Puesto que el alumnado, a medida que va avanzando en su camino académico no se para a pensar demasiado sobre su futuro profesional y cuando se acerca ese momento le surgen muchas dudas, consideramos que es preciso que todo esto se trabaje desde cursos iniciales y así el alumnado sea más consciente de que van a tener que elegir un camino del que puede que dependa su futuro.

En esta unidad también puede participar el alumno que presenta dislexia, ya que puede acceder a la realización de cada una de las actividades, con el fin de conseguir una completa inclusión del alumnado.

Ejemplo de algunas de las actividades que se llevarán a cabo en esta unidad de intervención:

La primera sesión va a constar de una actividad en la cual se plantea que los alumnos reflexionen sobre las actividades que más le gustan y así descubrirán la inteligencia donde tienen más afinidad.

Tabla 4

Actividad 1 de la primera sesión

\section{Actividad 1: Qué inteligencia se me da mejor}

\section{Temporalización}

- 50 minutos.

\section{Objetivo/s específicos}

- Profundizar en el conocimiento de las destrezas escolares así como su relación 
con las Inteligencias Múltiples.

- Estimular la toma de decisiones acerca de las habilidades seleccionadas.

\section{Contenidos}

- Profundiza en el conocimiento de las destrezas escolares así como su relación con las Inteligencias Múltiples.

- Estimula la toma de decisiones acerca de las habilidades seleccionadas.

\section{Desarrollo de la actividad}

A través de un listado de actividades, agrupadas por Inteligencias Múltiples, el alumnado tiene que valorar cuál de las actividades se les da mejor y así conocerá cual es la inteligencia donde tiene más habilidad. Después tiene que ordenarlas empezando por la que tiene más facilidad. Hay que explicarle al alumnado que esta actividad le va a ser útil para tomar decisiones en cuanto a las asignaturas optativas que tiene que escoger en el próximo curso y así ir guiando su camino académico y profesional (Anexo 2).

\section{Recursos y materiales}

- Lápiz, goma y anexo 2.

\section{Criterios de evaluación}

- Colabora en el conocimiento de la relación existente entre las destrezas escolares y las Inteligencias Múltiples.

- Toma decisiones sobre cuáles son sus habilidades más destacadas.

\begin{tabular}{c|cc}
\hline \multicolumn{1}{c|}{ Rol del profesor } & \multicolumn{1}{c}{ Organización del espacio } \\
\hline$\bullet \quad \begin{array}{l}\text { Orientador y guía, } \\
\text { asegurándose que la actividad } \\
\text { la realiza adecuadamente. }\end{array}$ & $\bullet \quad$ En el aula ordinaria. \\
\hline
\end{tabular}

Fuente: elaboración propia

\section{Resultados}

\section{Evaluación de los resultados del programa de orientación e intervención psicopedagógica}

Para la evaluación de los resultados vamos a realizar dos procesos fundamentales, uno relacionado con el proceso de enseñanza-aprendizaje del alumnado y otro con la práctica docente.

Por un lado, la evaluación del proceso de enseñanza-aprendizaje del alumnado se llevará a cabo con la revisión ordinaria, por parte del tutor, de los resultados obtenidos en cada una de las sesiones de trabajo. De esta manera, se observará si el programa de intervención ha tenido efecto en los estudiantes o por el contrario no han tenido buenos resultados y no les ha ayudado en su progreso.

Por otro lado, el profesorado encargado de la aplicación del programa de intervención, mediante un cuestionario online (Anexo 3), se encargará de evaluar la metodología utilizada, cómo se atiende a la diversidad, la planificación. Así sabremos cuáles son los puntos de flaqueza que puede tener el programa y mejorarlos, además de saber también cuáles han sido los aspectos positivos del mismo. 
Estas evaluaciones serán revisadas por el orientador del centro. Con estas evaluaciones pretendemos poder mejorar todo aquello que haga que no funcione el programa correctamente y así poder ofrecer un programa de calidad con una atención adaptada a las necesidades del alumno.

\section{Toma de decisiones}

En el caso de que la unidad de intervención resulte exitosa, se determinará poder llevarla a cabo en los demás cursos de la Educación Secundaria Obligatoria. Con ello, se potenciará el uso de nuevas metodologías de trabajo así como la introducción de la Teoría de las Inteligencias Múltiples en el proceso de enseñanza-aprendizaje tanto de alumnos que no presenten necesidades específicas de apoyo educativo como en aquellos que si las presenten, consiguiendo que haya una total inclusión del alumnado.

Sin embargo, si los resultados fueran negativos y tanto los alumnos como los profesores no alcanzaran los objetivos propuestos, se tendrían que tomar medidas como mejorar la metodología planteada o el contenido de la unidad. Además se llevarían evaluaciones tanto al alumnado como al profesorado para localizar los puntos más débiles del programa y así poder modificarlos. De este modo, se intentará responder a las necesidades del alumnado de un modo adecuado.

\section{Conclusiones}

Con este proyecto de intervención tratamos de ayudar al alumnado a que pueda conocerse tanto a sí mismo como a los demás, que tenga autonomía, que sea capaz de trabajar en grupo respetando las decisiones de los demás, que sepa tomar decisiones, así como el descubrimiento de nuevas formas de trabajo que haga más dinámico su proceso de enseñanzaaprendizaje.

Mediante el uso de las Inteligencias Múltiples, trabajadas desde diferentes perspectivas en cada una de las unidades de intervención, hemos querido conseguir un desarrollo integral del alumnado y además ayudar al profesorado a conocerlo y así poder solventar todas sus necesidades. Con este tipo de trabajo, tratamos de conseguir que el alumnado sea consciente de que existen diferentes tipos de inteligencias y que a través de su estimulación, van a conocer la inteligencia donde pueden tener más o menos habilidad, ya que a través de la combinación de las distintas inteligencias podrán tener un mejor progreso en su aprendizaje.

Por otro lado, este conocimiento de la Teoría de las Inteligencias Múltiples lo hemos querido llevar a cabo por medio de metodologías activas, como es el trabajo cooperativocolaborativo. De esta forma, el alumnado trabaja en grupo y desarrolla sus habilidades sociales y personales, complementan su aprendizaje de forma conjunta aprendiendo a escuchar a los compañeros, con lo cual no solo respetan su aprendizaje sino también el del resto del grupo, teniendo una evolución del conocimiento de forma constructiva y participativa.

Para finalizar, con este planteamiento de trabajo, hemos querido tener en cuenta la inclusión de todo el alumnado, y esto lo hemos llevado a cabo por medio de la combinación de las Inteligencias Múltiples y las dificultades de aprendizaje. Hemos considerado que con esta nueva forma de trabajo, podemos potenciar el interés, la motivación por aprender de este alumnado y mediante el trabajo cooperativo-colaborativo formar grupos heterogéneos donde puedan complementarse mutuamente. 


\section{Referencias}

Aguilar Calvo, M. (2011). Las dificultades de aprendizaje: diagnóstico e intervención. Madrid: Editorial CEP.

Andrade, P. (2009). Dificultades en el aprendizaje (Inteligencias Múltiples). Recuperado de http://inteligenciasmultiplespnl.blogspot.com.es/2009/06/dificultades-en-elaprendizaje.html.

Antunes, C. (2001). Estimular las inteligencias múltiples. Qué son. Cómo se manifiestan. Cómo funcionan. Madrid: Narcea.

De la Cruz García, P., y García Tíscar, E. (2017). Formar al profesorado para conseguir centros educativos inclusivos: creando redes. En A. Rodríguez-Martín (Comp.), Prácticas Innovadoras inclusivas: retos y oportunidades, 349-356. Oviedo: Universidad de Oviedo.

Gardner, H. (2001). Estructuras de la Mente. La Teoría de Las Inteligencias Múltiples. Colombia: Fondo de la Cultura Económica LTDA.

Gardner, H., Komhaber, M., y Krechevsky, M. (1993). Abordar el concepto de Inteligencia. En H.Gardner (Coord). Intetigencias múltiples: lit teoría en lal'ráctica, 243-259. Barcelona: Paidós Ibérica.

Gomis Selva, N. (2007). Evaluación de las inteligencias múltiples en el contexto educativo a través de expertos, maestros y padres (tesis doctoral inédita). Universidad de Alicante.

González Muñoz, D. (2014). Inteligencias múltiples y dificultades de aprendizaje. Padres y Maestros, (357), 10-14. doi: http://dx.doi.org/10.14422/pym.v0i357.3291.

Huelmo García, J., y Fernández Hawrylak, M. (2017). Competencias profesionales del docente en la comunidad de Castilla y León para dar cumplimiento al principio de inclusión. En A. Rodríguez-Martín (Comp.). Prácticas Innovadoras inclusivas: retos y oportunidades, 249-256. Oviedo: Universidad de Oviedo.

Instrucciones de 8 de marzo de 2017, de la dirección general de participación y equidad, por las que se actualiza el protocolo de detección, identificación del alumnado con necesidades específicas de apoyo educativo y organización de la respuesta educativa. Sevilla.

LEY 17/2007, de 10 de diciembre, de Educación de Andalucía. (BOJA, No 252, 26 de diciembre de 2007).

Ley Orgánica 8/2013, de 9 de diciembre, para la mejora de la calidad educativa. (BOE, $\mathrm{N}^{\circ}$ 295, 10 de diciembre de 2013).

Llanos Días, S. (2006). Dificultades de aprendizaje. Centro de Estudios Sociales y Publicaciones (CESIP). Recuperado de http://www.cesip.org.pe/sites/default/files/27dificultades_de_aprendizaje.pdf.

Matamoros, A. M. A. (1999). Inteligencias múltiples: Una aproximación a la teoría de Howard Gardner. Horizontes Pedagógicos, 1(1), 19-27.

Molejón Asenjo, L., y Fernández-Viciana, A. (2017). Todos somos diversos y todos somos capaces: las inteligencias múltiples y su influencia en la autoeficacia. En A. 
Rodríguez-Martín (Comp.). Prácticas Innovadoras inclusivas: retos y oportunidades, 611-618. Oviedo: Universidad de Oviedo.

Monedero, C. (1989). Dificultades de Aprendizaje Escolar: Una Perspectiva Neuropsicológica. Madrid: Pirámide.

Orden ECD/65/2015, de 21 de enero, por la que se describen las relaciones entre las competencias, los contenidos y los criterios de evaluación de la educación primaria, la educación secundaria obligatoria y el bachillerato. (BOE, No25, 29 de enero de 2015).

Pérez Solis, M. (2002). Orientación Educativa y dificultades de aprendizaje. Madrid: Paraninfo.

Salmerón Vílchez, P. (2002). Evolución de los conceptos sobre inteligencia. Planteamientos actuales de la inteligencia emocional para la orientación educativa. Educación, XX1 (5), 97-121.

Ugalde, K. (s.f.). El aprendizaje y las Inteligencias Múltiples. Contigo Salud vivir mejor. Recuperado de: http://www.contigosalud.com/el-aprendizaje-y-las-inteligenciasmultiples.

Varela, C., y Cruz, I. (2006). El Proyecto Spectrum: aplicación y actividades de aprendizaje de ciencias en el primer ciclo de la Educación Primaria. Revista de Educación, (339), 947-958. 
Anexos

Anexo 1: Buscamos palabras y formamos números

Tabla 5

Actividad 2 de la segunda sesión

\begin{tabular}{|c|c|c|c|}
\hline $\begin{array}{c}\text { Palabras } \\
\text { desordenadas }\end{array}$ & $\begin{array}{c}\text { Palabras } \\
\text { ordenadas }\end{array}$ & $\begin{array}{l}\text { Número de } \\
\text { sílabas }\end{array}$ & Números \\
\hline za-da-man-na & Manzana & 3 & \multirow{4}{*}{$\begin{array}{l}\text { Inventa un número, } \\
\text { con el número de } \\
\text { sílabas de estas } \\
\text { cuatro palabras. } \\
\text { 3.433 }\end{array}$} \\
\hline $\begin{array}{l}\text { tra-do-dra- } \\
\text { cua }\end{array}$ & Cuadrado & 3 & \\
\hline ta-dor-je-tu-a & Tatuaje & 4 & \\
\hline sol-dor-ca-se & Secador & 3 & \\
\hline vi-rra-ta-gui & & & \multirow[t]{3}{*}{$\begin{array}{l}\text { Inventa un número, } \\
\text { con el número de } \\
\text { sílabas de estas tres } \\
\text { palabras. }\end{array}$} \\
\hline bo-ce-ca-dl-Ilo & & & \\
\hline $\begin{array}{l}\text { se-tan-rí-a-es- } \\
\text { te }\end{array}$ & & & \\
\hline $\begin{array}{l}\text { ven-cor-ti-lar- } \\
\text { dor }\end{array}$ & & & \multirow[t]{2}{*}{$\begin{array}{l}\text { Inventa un número, } \\
\text { con el número de } \\
\text { sílabas de estas dos } \\
\text { palabras. }\end{array}$} \\
\hline Bo-ya-te-lla & & & \\
\hline
\end{tabular}

Tabla 6

Descomposición de los números

Descomposión de los números

\begin{tabular}{l|l|l|l|l}
\hline Número & Unidad de millar & Centena & Decena & Unidad \\
\hline 3.433 & 3 & 4 & 3 & 3 \\
\hline & & & & \\
\hline
\end{tabular}




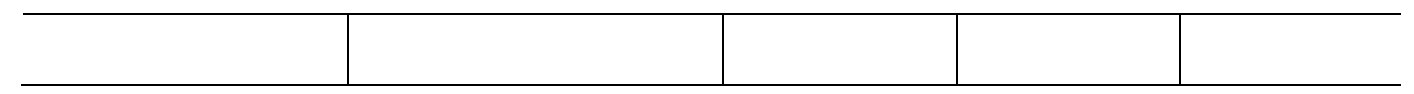

Nota: Fuente: elaboración propia

Anexo 2: Qué inteligencia se me da mejor

Tabla 7

Actividad 1 de la primera sesión

\begin{tabular}{|c|c|c|c|}
\hline \multicolumn{4}{|c|}{ Qué inteligencia se me da mejor } \\
\hline $\mathbf{X}$ & Actividades & Destreza & $\begin{array}{l}\text { Inteligencias } \\
\text { múltiples }\end{array}$ \\
\hline & Leer & \multirow{5}{*}{ Para el lenguaje } & \multirow{5}{*}{$\begin{array}{l}\text { Inteligencia } \\
\text { Lingüística }\end{array}$} \\
\hline & $\begin{array}{l}\text { Escribir historias, poemas, } \\
\text { biografías }\end{array}$ & & \\
\hline & Redactar comentarios & & \\
\hline & Dar sugerencias & & \\
\hline & Buscar en la biblioteca & & \\
\hline & $\begin{array}{l}\text { Resolver operaciones } \\
\text { matemáticas }\end{array}$ & \multirow{3}{*}{$\begin{array}{l}\text { Para las } \\
\text { matemáticas }\end{array}$} & \multirow{3}{*}{$\begin{array}{l}\text { Inteligencia } \\
\text { matemática }\end{array}$} \\
\hline & $\begin{array}{l}\text { Resolver problemas numéricos } \\
\text { con medidas, datos, cantidades, } \\
\text { distancias planteando } \\
\text { estrategias. }\end{array}$ & & \\
\hline & Usar fórmulas matemáticas & & \\
\hline & Cantar & \multirow{5}{*}{ Para la música } & \multirow{5}{*}{ Inteligencia musical } \\
\hline & Solfear & & \\
\hline & Afinar el sonido, & & \\
\hline & Tener ritmo & & \\
\hline & Tocar un instrumento & & \\
\hline & Discriminar detalles & \multirow{7}{*}{$\begin{array}{l}\text { Para la expresión } \\
\text { plástica-visual. }\end{array}$} & \multirow{7}{*}{$\begin{array}{l}\text { Inteligencia visual- } \\
\text { espacial }\end{array}$} \\
\hline & Dibujar & & \\
\hline & Realizar mapas & & \\
\hline & Representar recuerdos & & \\
\hline & Crear álbumes para fotos & & \\
\hline & Diseñar objetos & & \\
\hline & Comprender dibujos & & \\
\hline & $\begin{array}{l}\text { Utilizar los movimientos del } \\
\text { cuerpo para expresar emociones }\end{array}$ & \multirow{4}{*}{ Habilidades físicas } & \multirow{4}{*}{$\begin{array}{l}\text { Inteligencia } \\
\text { corporal-cinestética }\end{array}$} \\
\hline & Crear juegos, simulaciones & & \\
\hline & Bailar & & \\
\hline & Hacer deporte & & \\
\hline & Animales, insectos, plantas & \multirow{4}{*}{ Científicas } & \multirow{4}{*}{$\begin{array}{l}\text { Inteligencia } \\
\text { naturalista }\end{array}$} \\
\hline & Juegos científicos & & \\
\hline & $\begin{array}{l}\text { Utilizar los objetos del } \\
\text { laboratorio }\end{array}$ & & \\
\hline & Investigación & & \\
\hline & Crear grupos y trabajar de & & \\
\hline
\end{tabular}


Inteligencias múltiples y dificultades de aprendizaje

\begin{tabular}{l|l|l|l}
\hline & forma cooperativa & \multirow{2}{*}{ Relaciones sociales } & $\begin{array}{l}\text { Inteligencia } \\
\text { interpersonal }\end{array}$ \\
\hline & $\begin{array}{l}\text { Enseñar, ayudar a otras } \\
\text { personas }\end{array}$ & \\
\hline Asumir diferentes roles & \multirow{2}{*}{ Filosófica } & $\begin{array}{l}\text { Inteligencia } \\
\text { intrapersonal }\end{array}$ \\
\hline & Estudiar diferentes culturas & \\
\hline & Describir tus propias cualidades & \\
\hline & Reflexionar silenciosamente & Ser independiente & Inteligencias Múltiples \\
\hline \multicolumn{2}{|c|}{ Ordenar las destrezas empezando por la que tengas más facilidad } \\
\hline \multicolumn{2}{|c|}{ Destrezas } & \\
\hline
\end{tabular}

Nota: Fuente: elaboración propia

Anexo 3: Acceso al cuestionario

Tabla 8

Cuestionario

\section{EVALUACIÓN PARA EL PROFESORADO}

Esta evaluación se va a llevar a cabo para poder obtener una conclusión final de la puesta en marcha del programa de intervención psicopedagógica. Indique su grado (de 1 a 4) de acuerdo con las afirmaciones que se realizan sobre los contenidos que aparecen en el programa.

\begin{tabular}{|c|c|c|c|}
\hline 1 & 2 & 3 & 4 \\
\hline Totalmente en desacuerdo & Poco de acuerdo & De acuerdo & Totalmente de acuerdo \\
\hline
\end{tabular}

\section{ACCESO AL CURRÍCULO UNIVERSITARIO}

\begin{tabular}{|l|r|}
\hline 1. ¿Han sido útiles los contenidos impartidos? & 1234 \\
\hline 2. ¿Los contenidos propuestos se han adaptado a las necesidades del alumnado? & 1234 \\
\hline 3. ¿Se han alcanzado los objetivos planteados? & 1234 \\
\hline 4. ¿Ha sido complicado impartir la clase con la nueva metodología? & 1234 \\
\hline 5. ¿Se han adaptado los alumnos a la nueva forma de trabajo? & 1234 \\
\hline 6. ¿Has notado si los alumnos han mejorado en su forma de aprendizaje? & 124 \\
\hline 7. ¿Ha habido comunicación con el resto de tutores implicados en el programa? & 1234 \\
\hline 8. ¿Piensas que se llega hacer inclusión con todo el alumnado? & 1234 \\
\hline 9. ¿Crees que es afectiva su aplicación? & 1234 \\
\hline
\end{tabular}


10. Qué puntos te resultan más interesantes

11. Qué puntos crees que se deberían mejorar

Nota: Fuente: elaboración propia

Fecha de recepción: 05/10/2017

Fecha de revisión: 09/10/2017

Fecha de aceptación: 06/11/2017 